\title{
Relación entre Índice Facial Superior e Índice Nasal en Cráneos Chilenos Adultos
}

\author{
Relation between Superior Facial Index and Nasal Index in Chilean Adult Crania
}

"María Fabiola Bustamante; *Ramón Fuentes; *Tania Flores \& **Antonio Sanhueza

BUSTAMANTE, M. F.; FUENTES, R.; FLORES, T. \& SANHUEZA, A. Relación entre índice facial superior e índice nasal en cráneos chilenos adultos. Int. J. Morphol., 29(3):810-815, 2011.

RESUMEN: La cara es el sello principal de la identidad, lo que nos define como individuos en los contactos interpersonales. Las características métricas aportadas por la craneometría nos permiten caracterizar cráneos y caras por medio de los índices. Se realizó un estudio craneométrico en 32 cráneos de individuos adultos de ambos sexos. En ellos se midieron diámetros faciales y se determinaron sus índices según Bidegain \& Carvalho de Mello. La altura facial superior (n-pr) promedio fue de 66,20mm (DE $\pm 5,26)$, con límite máximo de 76,68mm y mínimo de 51,22mm. El ancho facial máximo (zy-zy) promedio fue de 127,05mm (DE $\pm 6,85$ ), con límite máximo $139,08 \mathrm{~mm}$ y mínimo de $112,77 \mathrm{~mm}$. El ancho nasal (al-al) promedio fue de 23,99 mm (DE $\pm 2,81$ ), con límite máximo de un 35,13mm y mínimo de 18,97 mm. La altura nasal (n-ns) promedio fue de 50,97 mm (DE $\pm 3,58$ ), con límite máximo de 55,96 mm y mínimo de 39,58mm. El Índice Facial Superior promedio fue de 52,20 (DE \4,54), con límite máximo de 62,29 y mínimo de 43,92 determinando la tendencia meseno en cráneos de género masculino y femenino. El Índice Nasal promedio fue de 47,30 (DE $\pm 6,28)$ con límite máximo de 67,14 y mínimo de 36,23 determinando características leptorrinas tanto en cráneos masculinos como femeninos. Los datos obtenidos permitirán a los profesionales del área salud contar con nuevos antecedentes para estudios antropométricos y antropológicos.

PALABRAS CLAVE: Craneometría; Índice facial superior; Índice Nasal.

\section{INTRODUCCIÓN}

Osteometría es el estudio y análisis de restos humanos esqueletizados, que utiliza métodos homogenizados y estandarizados para la observación de características métricas y no-métricas, con el fin de registrar la forma y el tamaño del hombre en su variabilidad cronológica y geográfica. Los estudios craneométricos nos ayudan a conocer la forma de los cráneos. La aplicación de métodos, mediciones e instrumentos en la craneometría permite la cuantificación y la comparación de los resultados de las investigaciones. La utilización de datos objetivos obtenidos a través de la craneometría asumen un rol decisivo en la identificación individual en cuanto a constitución, sexo, raza y edad de los individuos (Vanrell \& Borborema, 1953).

A lo largo de la historia la simetría facial siempre ha sido un tema de interés anatómico y artístico y como indicara Fernández et al. (2001), la cara es el sello principal de la identidad personal, es decir, lo que nos define como individuos en los contactos interpersonales (del Sol, 2006). Los humanos nos comunicamos a través del rostro y para conseguir una evaluación facial más objetiva, durante mucho tiempo fueron practicados estudios métricos. Andreas Vesalius (1514-1564), en sus primeros estudios en cadáveres y en cráneos de hombres de la época describe cómo todas sus estructuras tienen características simétricas (Vallejo-Manzur et al., 2003). El concepto artístico de Leonardo Da Vinci (1452 -1519), considera que el rostro del ser humano debe ser simétrico para poder tener armonía y belleza (Naini et al., 2006). De acuerdo a los estudios de Enlow (1990) la base craneal determina las características dimensionales, angulares y topográficas de la cara; en tanto, las características métricas aportadas por la craneometría, nos permiten obtener información suficiente para caracterizar y clasificar cráneos y caras por medio de los índices.

Entre los análisis más utilizados para el estudio de relaciones transversales que establecen los componentes esqueléticos y dentoalveolares de la cabeza, se encuentran

\footnotetext{
* Departamento de Odontología Integral, Facultad de Medicina, Universidad de La Frontera, Temuco, Chile.

*** Departamento de Matemáticas y Estadísticas, Facultad de Ingeniería, Ciencias y Administración, Universidad de La Frontera, Temuco, Chile.
} 
los análisis frontales, siendo el de Ricketts el más ampliamente utilizado (Grummons \& Kappeyne van de Coppello, 1987; Gregoret, 1997). Snodell et al. (1993) analizaron las relaciones craneales verticales y transversales en 25 hombres y 25 mujeres de raza blanca de 4 a 25 años de edad, determinando que las proporciones entre las mediciones transversales del esqueleto facial a los 6 años de edad fueron similares a las medidas de la edad adulta, no ocurriendo lo mismo con las medidas verticales. Ramos et al. (2007) analizaron las relaciones transversales faciales de 45 niños mesofaciales de la Región del Maule, Chile, mediante un análisis cefalométrico frontal en que se analizaron las dimensiones: ancho transpalatino, ancho maxilar, ancho facial y ancho mandibular. Las dimensiones encontradas fueron superiores a las descritas en la literatura. Se encontró que el ancho transpalatino mostraba una mediana correlación con el ancho maxilar, no así con el ancho facial. Kasai et al. (1995) y Ferrario et al. (1997) sugieren la existencia de una correlación entre craneometría y morfología maxilofacial y agregan que los individuos dolicocefálicos tienden a presentar un Índice Facial Morfológico más bien alargado (leptoprosopo) y los individuos braquicefálicos uno de tipo ancho (euriprosopo); del mismo modo sugieren que en un individuo dolicocéfalo se espera encontrar un Índice Facial Superior de tipo lepteno, en tanto en un individuo braquicéfalo la tendencia es de tipo eurieno. Estudios de Lehmann \& Marquer (1960) acerca de la distribución del Índice Facial Superior según las categorías lepteno, meseno y eurieno en indios del grupo Guambiano-kokonuko, arrojaron como resultado un franco predominio de la categoría meseno. Oliver et al. (1996b), realizaron un estudio en diversos grupos de indochinos en los cuales se determinó un Índice Facial meseno para todos ellos. Anh \& Tien-Loi (1966) estudiaron el Índice Nasal en individuos Rhades hallando una distribución similar de las categorías mesorrino y camerino en la muestra estudiada.

Estas informaciones son de importancia tanto para anatomistas, antropólogos físicos, odontólogos y cirujanos maxilofaciales. Este estudio tiene por finalidad caracterizar una muestra de cráneos chilenos, según su Índice Facial Superior (IFS) e Índice Nasal (IN) a través de la medición de sus diámetros faciales.

\begin{tabular}{llr}
\hline Índice & & $\%$ \\
\hline Facial Superior (IFS) & Hipereurieno (cara muy ancha) & $\mathrm{X}-44,9$ \\
& Eurieno (cara ancha) & $45,0-49,0$ \\
& Meseno (cara mediana) & $50,0-54,9$ \\
& Lepteno (cara larga) & $55,0-59,9$ \\
& Hiperlepteno (cara muy larga) & $60,0-\mathrm{X}$ \\
& & \\
& Leptorrino (nariz larga) & $\mathrm{X}-46,9$ \\
& Mesorrino (nariz mediana) & $47,0-50,9$ \\
& Camerrino (nariz ancha) & $51,0-57,9$ \\
& Hipercamerrino (nariz muy ancha) & $58,0-\mathrm{X}$ \\
\hline
\end{tabular}

\section{MATERIAL Y MÉTODO}

El estudio se realizó en 32 cráneos chilenos de individuos adultos de ambos sexos (24 masculinos y 8 femeninos) pertenecientes a la colección del Departamento de Anatomía de la Pontificia Universidad Católica de Santiago, Chile. Cabe destacar que uno de los cráneos en estudio presentaba fractura a nivel de Prosthion (pr), motivo que imposibilitó la medición de la altura facial superior en él, sin embargo se consideró para todas las demás mediciones realizadas en el estudio. Se midió el IFS e IN según Bidegain \& Carvalho de Mello (1979). Los puntos craneométricos necesarios para obtener dichos índices, fueron los siguientes:

Nasion (n): Punto mediano de encuentro entre las suturas internasal y frontonasal.

Prosthion (pr): Punto mediano más anterior en el proceso alveolar del maxilar, entre los incisivos centrales.

Zygion (zy): Punto paramediano más lateral del proceso cigomático. Nasospinale (ns): Punto mediano ubicado en el margen inferior de la espina nasal anterior, también denominado Subnasale o Nasale. Alar (al): Punto paramediano localizado en la parte más lateral de la apertura piriforme.

El cálculo del IFS se realizó de acuerdo a la siguiente fórmula:

$$
\mathrm{IFS}=\frac{\text { distancia } \mathrm{n}-\mathrm{pr} \text { (altura facial superior) }}{\text { distancia zy-zy (ancho facial máximo) }} \mathrm{X} 100
$$

El cálculo del IN se realizó de acuerdo a la siguiente fórmula:

$$
\mathrm{IN}=\frac{\text { distancia al-al (ancho nasal) }}{\text { distancia n-ns (altura nasal) }} \times 100
$$

Las mediciones fueron realizadas con ayuda de un compás de espesor para la distancia zy-zy y para las otras mediciones, utilizamos un compás digital de corredera (cáliper). La clasificación de los índices IFS e IN fue realizada según Bidegain \& Carvalho de Mello (Tabla I).

Tabla I. Clasificación de los índices IFS e IN fue realizada según Bidegain \& Carvalho de Mello (1979). 


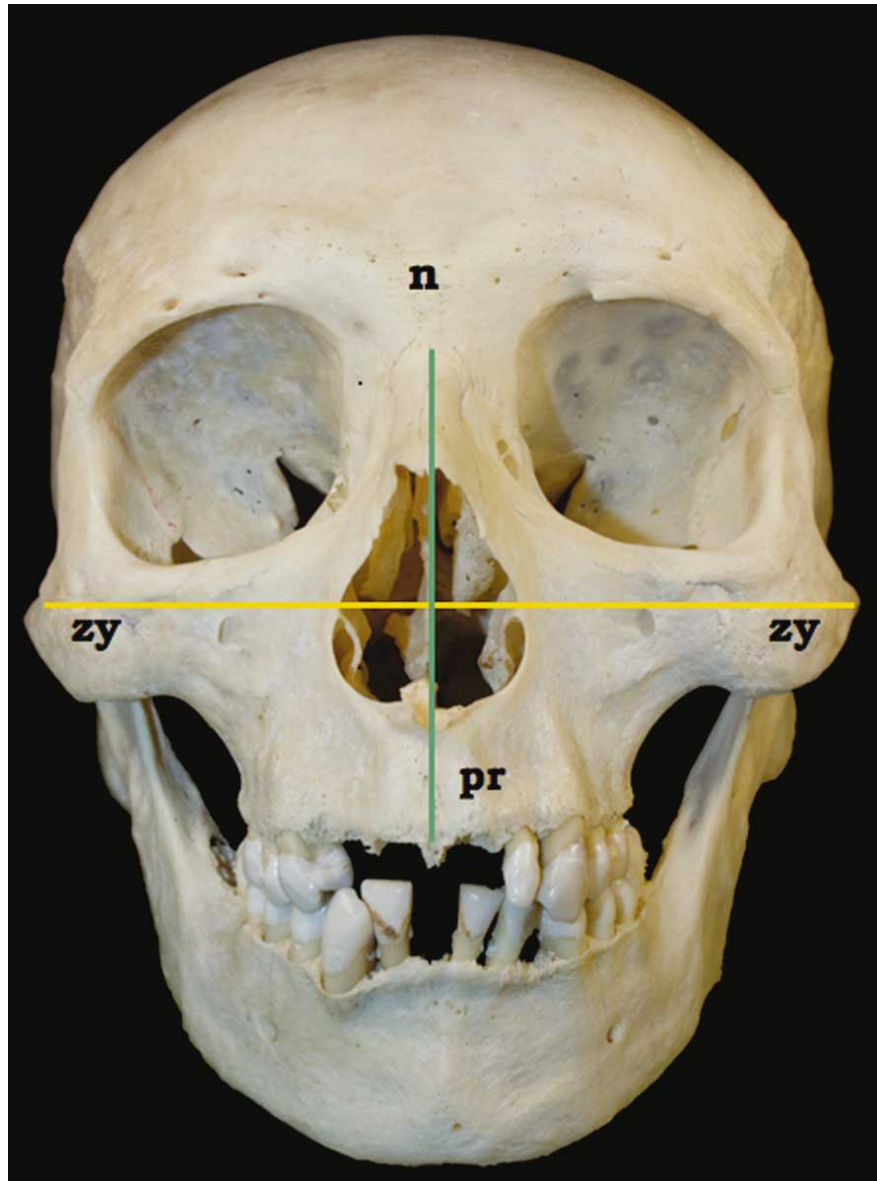

Fig. 1. En verde la altura facial superior (n-pr), en amarillo: Ancho facial máximo (zy-zy).

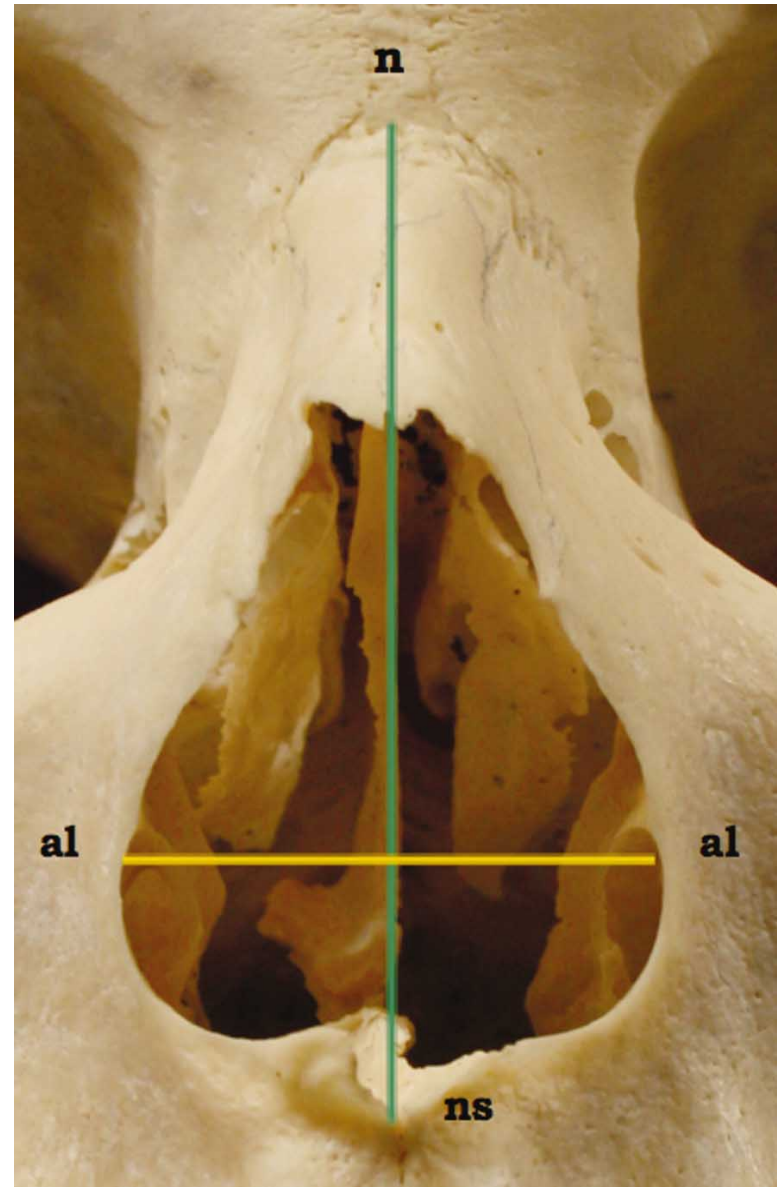

Fig. 2: En amarillo el ancho nasal (al-al), en verde: Altura nasal (n-ns).
Se realizó análisis estadístico descriptivo por medio de tablas, promedios y desviación standar; además se realizó estudio estadístico inferencial a través de la prueba estadística Chi Cuadrado.

\section{RESULTADOS}

Los registros de las medidas de altura facial superior (n-pr), ancho facial máximo (zy-zy) e IFS y medidas ancho nasal (al-al), altura nasal (n-ns) e IN con sus respectivos promedios, desviación estándar, valores mínimos y máximos fueron obtenidos de 32 cráneos adultos de ambos sexos, de la Pontificia Universidad Católica de Chile, Santiago, Chile, datos que se resumen en las Tablas II y III

Tabla II. Medidas de altura facial superior (n-pr), ancho facial máximo (zy-zy) e IFS.

\begin{tabular}{llcccc}
\hline & & Promedio & Desviación Estandar & Mínimo & Máximo \\
\hline Hombre & n-pr $(\mathbf{m m})$ & 67,57 & 4,14 & 60,19 & 76,68 \\
& zy-zy (mm) & 127,50 & 6,79 & 112,77 & 139,08 \\
& IFS (\%) & 52,37 & 4,57 & 44,81 & 62,29 \\
Mujer & n-pr (mm) & 65,11 & 8,03 & 51,22 & 74,21 \\
& zy-zy (mm) & 125,72 & 7,31 & 115,30 & 135,84 \\
& IFS (\%) & 51,70 & 4,73 & 43,92 & 55,62 \\
Total & n-pr $(\mathbf{m m})$ & 66,20 & 5,26 & 51,22 & 76,68 \\
& zy-zy (mm) & 127,05 & 6,85 & 112,77 & 139,08 \\
& IFS (\%) & 52,20 & 4,54 & 43,92 & 62,29 \\
\hline
\end{tabular}


Tabla III. Medidas de ancho nasal (al-al), altura nasal (n-ns) e IN, en 32 cráneos adultos de ambos sexos, de la Pontificia Universidad Católica de Chile, Santiago, Chile.

\begin{tabular}{llcccc}
\hline & & Promedio & Desviación Estandar & Mínimo & Máximo \\
\hline Hombre & al-al (mm) & 23,86 & 1,94 & 19,74 & 27,17 \\
& n-ns (mm) & 51,34 & 2,88 & 46,08 & 55,79 \\
\multirow{3}{*}{ Mujer } & IN (\%) & 46,58 & 4,32 & 38,43 & 53,66 \\
& al-al (mm) & 24,42 & 4,71 & 18,97 & 35,13 \\
& n-ns (mm) & 49,82 & 5,24 & 39,58 & 55,55 \\
\multirow{3}{*}{ Total } & IN (\%) & 49,46 & 10,30 & 36,23 & 67,14 \\
& al-al (mm) & 23,99 & 2,81 & 18,97 & 35,13 \\
& n-ns (mm) & 50,97 & 3,58 & 39,58 & 55,69 \\
& IN (\%) & 47,30 & 6,28 & 36,23 & 67,14 \\
\hline
\end{tabular}

Tabla IV. Distribución de Índice Facial Superior según sexo. p=0,6862

\begin{tabular}{lcccc}
\hline & Eurieno & Lepteno & Meseno & Total \\
\hline Hombre & $7(29,17 \%)$ & $7(29,17 \%)$ & $10(41,67 \%)$ & $24(100 \%)$ \\
Mujer & $1(14,29 \%)$ & $2(28,57 \%)$ & $4(57,14 \%)$ & $7(100 \%)$ \\
& 8 & 9 & 14 & 31 \\
\hline
\end{tabular}

Tabla V. Distribución de Índice Nasal según sexo.

\begin{tabular}{lcccc}
\hline & Camerrino & Leptorrino & Mesorrino & Total \\
\hline Hombre & $6(25,00 \%)$ & $13(54,17 \%)$ & $5(20,83 \%)$ & $24(100 \%)$ \\
Mujer & $2(25,00 \%)$ & $4(50,00 \%)$ & $2(25,00 \%)$ & $8(100 \%)$ \\
& 8 & 17 & 7 & 32 \\
\hline $\mathrm{p}=0,9669$ & & & &
\end{tabular}

Tabla VI. Distribución de Índice Nasal según Niveles de Índice Facial.

\begin{tabular}{lcccc}
\hline & Camerrino & Leptorrino & Mesorrino & Total \\
\hline Eurieno & $5(62,50 \%)$ & $1(12,50 \%)$ & $2(25,00 \%)$ & $8(100 \%)$ \\
Lepteno & $1(11,11 \%)$ & $7(77,78 \%)$ & $1(11,11 \%)$ & $9(100 \%)$ \\
Meseno & $2(14,29 \%)$ & $9(64,29 \%)$ & $3(21,43 \%)$ & $14(100 \%)$ \\
& 8 & 17 & 6 &
\end{tabular}

respectivamente, donde podemos observar que el valor promedio de la altura facial superior (n-pr) en los cráneos de sexo masculino fue de 67,57 mm (DE $\pm 4,14$ ), la misma variable en los cráneos femeninos fue de 65,11 mm (DE \pm $8,03)$.

El ancho facial máximo (zy-zy) en los cráneos de sexo masculino fue de $127,50 \mathrm{~mm}$ ( $\mathrm{DE} \pm 6,79$ ), la misma variable en los cráneos femeninos fue de 125,72mm (DE $\pm 7,31$ ). El ancho nasal (al-al) en los cráneos de sexo masculino fue de $23,86 \mathrm{~mm}$ (DE $\pm 1,94$ ), la misma variable en los cráneos femeninos fue de 24,42mm (DE $\pm 4,71$ ). La altura nasal (n-ns) en los cráneos de sexo masculino fue de 51,34mm (DE $\pm 2,88)$, la misma variable en los cráneos femeninos fue de 49,82mm (DE $\pm 5,24$ ). El IFS promedio fue de un $52,37 \%$ en cráneos masculinos y un $51,70 \%$ en cráneos femeninos.
La distribución numérica y porcentual del IFS según sexo se observan en la Tabla IV, registrándose un mayor porcentaje de casos en la categoría de la variable meseno tanto para cráneos masculinos, como femeninos con un $41,67 \%$ y $57,14 \%$ respectivamente. La distribución numérica y porcentual del IN según sexo se observa en la tabla V, registrándose un mayor porcentaje de casos en la categoría leptorrino alcanzando un valor del $54,17 \%$ para cráneos masculinos y de un 50,00\% para cráneos femeninos. La distribución numérica y porcentual según la clasificación de los índices IFS e IN la observamos en la tabla VI, donde se observa una distribución del Índice Nasal diferente para cada uno de los niveles del Índice Facial. A partir de esta información se encuentran tres asociaciones más prevalentes que en orden decreciente fueron Lepteno-Leptorrino (caras largas con nariz larga), luego Meseno-Leptorrino (caras medianas con nariz larga), seguida por la asociación Eurieno- 
Camerrino (caras anchas con nariz ancha). Mayores detalles de los hallazgos se encuentran en la sección tablas.

\section{DISCUSIÓN}

En la muestra en estudio el valor promedio de la altura facial superior en los cráneos de sexo masculino fue de $67,57 \mathrm{~mm}(\mathrm{DE} \pm 4,14)$, la misma variable en los cráneos femeninos alcanzó el valor promedio de $65,11 \mathrm{~mm}(\mathrm{DE} \pm 8,03)$. En cuanto al ancho facial máximo, el valor promedio total de los cráneos en estudio fue de 127,05mm ( $\mathrm{DE} \pm 6,85$ ), valor levemente más elevado al obtenido por Ramos et al. en su estudio realizado en pacientes de las clínicas odontológicas de la Universidad de Talca, Chile que arrojaron resultados de ancho facial máximo con valores promedios cercanos a $121,82 \mathrm{~mm}$.

El valor promedio para Índice Facial Superior (IFS) que alcanzó un $52,2 \%$ y el predominio general de la categoría meseno en cráneos de individuos de ambos sexos, se asemejan a los obtenidos por Gessain (1958) en su estudio realizado en un grupo de cráneos de esquimales de Angmassalik, de la Costa Este de Groenlandia, en quienes observó para el índice facial superior un valor promedio de un 50,9\% con predominio de la categoría meseno. Estudios de Lehmann \& Marquer en indios del grupo Guambianokokonuko, determinaron que el índice facial superior es francamente meseno y el valor promedio del índice facial superior de un 55,6\% levemente superior al valor obtenido en nuestro estudio $(52,20 \%)$. Por su parte, Olivier et al., (1966a), determinaron que los chinos del sur tenían un índice facial superior general meseno, similar a lo obtenido en nuestra muestra ya que la mayor parte de los cráneos examinados presentaban un índice facial superior de tipo meseno. En otro estudio de Oliver et al. (1996b), realizado en diversos grupos de indochinos se obtuvo como resultado el predominio del índice facial meseno para todos ellos.

Por otro lado, los valores del índice nasal general obtenidos en nuestro estudio fueron de un $47,30 \%$ (DE \pm $6,28)$ predominando la categoría leptorrino. Por su parte Anh \& Tien-Loi en individuos Rhades observaron la existencia de similares porcentajes de individuos mesorrinos y leptorrinos $(30,43 \%)$ y un $29,34 \%$ individuos camerinos, valores levemente superiores a los obtenidos en nuestro estudio. En nuestro estudio y en orden decreciente se presentaron las siguientes asociaciones estadísticamente significativas: lepteno-leptorrino; meseno-leptorrino; eurienocamerrino, siendo éstas las formas más comunes encontradas en la muestra estudiada.

La clasificación craneal de individuos adultos nos ayuda en la determinación de parámetros antropométricos, lo que será siempre muy valioso para el estudio de la evolución biológica del ser humano, su anatomía, su arte, su simetría permitiendo a los profesionales del área de la salud contar con nuevos antecedentes para futuros estudios.

\section{AGRADECIMIENTOS}

A los profesores titulares Hermes Bravo y Oscar Inzunza de la Pontificia Universidad Católica de Chile por facilitar el material de osteoteca, a la señora Karina Silva por la ayuda en la recolección de los datos, los alumnos Felipe Hein y Felipe Lemarie de la Universidad Austral de Chile, por su cooperación en la fotografía.

BUSTAMANTE, M. F.; FUENTES, R.; FLORES, T. \& SANHUEZA, A. Relation between superior facial index and nasal index in Chilean adult crania. Int. J. Morphol., 29(3):810-815, 2011.

SUMMARY: The face is the main symbol of identity which defines us as individuals as well as in the process of interpersonal contacts. Metric characteristics contributed by craniometry allow us to characterize crania and faces by means of indexes. A craniometric study was carried out in 32 crania of adult subjects of both sexes. Facial diameters were measured using indexes according to Bidegain \& Carvalho de Mello. Facial height average (n-pr) was $66.20 \mathrm{~mm}(\mathrm{SD} \pm 5.26)$ with maximum boundary of $76.68 \mathrm{~mm}$ and minimum of $51.22 \mathrm{~mm}$. Facial maximum width on average (zy - zy) was $127.05 \mathrm{~mm}$. (SD \pm 6.85 ), with maximum boundary of $139.08 \mathrm{~mm}$ and minimum of $112.7 \mathrm{~mm}$. Average Nasal width was $23.99 \mathrm{~mm}$ (SD \pm 2.81 ), with maximum boundary of $35.13 \mathrm{~mm}$ and minimum of $18.97 \mathrm{~mm}$. Nasal height average (n-ns) was $50.97 \mathrm{~mm}(\mathrm{SD} \pm 3.58)$ with maximum boundary of $55.96 \mathrm{~mm}$ and minimum of $39.58 \mathrm{~mm}$. Facial Superior Index on average was of 52.20 ( $\mathrm{SD} \pm 4.54)$ with maximum boundary of $62.29 \mathrm{~mm}$ and minimum of 43.92 determining the mesorrhine tendency in male and female crania. Nasal average index was 47.30 (SD \pm 6.28 ) with maximum boundary of 67.14 and minimum of 36.23 thereby determining leptorrhine characteristics in male as well as female crania. The information obtained will afford health care professionals access to new data for anthropometric and anthropological studies.

KEY WORDS: Craniometry; Facial top Index; Nasal Index. 


\section{REFERENCIAS BIBLIOGRÁFICAS}

Anh, T. \& Tien-Loi, V. Craniometrie des chinois du sud. Bull. et Mém. Soc. d’Anthrop. de Paris, 11(9):91-107, 1966.

Bidegain, P. C. \& Carvalho de Mello e Alvim, M. Manual para estudios Craneométricos y Craneoscópicos, 1979. Disponible en: http://www.cleber.com.br/ manual.pdf

Del Sol, M. Índices faciales en individuos mapuches. Int. J. Morphol., 24(4):587-90, 2006.

Enlow, D. H. Crecimiento maxilofacial. $3^{\text {a }}$ ed. México D. F., Interamericana, 1990.

Fernández, R. P.; Smyth, E.; Figueiras, A. \& Suárez, Q. D. Motivación psicosocial del paciente ortodóncico. Ortodoncia clínica, 4(1):34-8, 2001.

Ferrario, V.; Sforza, C.; Poggio, C.; Schmitz, J. H. \& Colombo, A. Soft tissue facial morphology related to headform: a three-dimensional quantitative analysis in childhood. J. Craniofac. Genet. Dev. Biol., 17(2):S 6-95, 1997.

Gessain, R. Les Eskimo d'Angmassalik principaux caracteres anthropologiques. L'Antropologie, 62(5-6): 452-84, 1958.

Gregoret, J. Ortodoncia y cirugía ortognática, diagnóstico y planificación de tratamiento. Barcelona, Publicaciones Médicas, 1997.

Grummons, D. C. \& Kappeyne van de Coppello, M. A A frontal asymmetry analysis. J. Clin. Orthod., 21(7): 448-65, 1987.

Kasai, K.; Moro, T.; Kanazawa, E. \& Iwasawa, T. Relationship between cranial base and maxillofacial morphology. Eur. J. Orthod., 17(5):403-10, 1995.

Lehmann, H. \& Marquer, P. Ètude anthropologique des indiens du groupe Guambiano- Kokonuko (Región de Popayán, Colombie). Bull. et Mém. Soc. d`Anthrop. De Paris, 11(1):177-240, 1960.

Naini, F.; Moss, J. \& Gill, D. The enigma of facial beauty: Esthetics, proportions, deformity, and controversy. Am. J. Orthod. Dentofacial Orthop., 130(3):277-82, 2006.
Olivier, G.; Phalippou, G. \& Tissier, H. Craniomètrie des chinois du sud. Bull. et Mém. Soc. d'Anthrop. de Paris, 11(9):55-66, 1966a.

Olivier, G.; Phalippou, G. \& Tissier, H. Craniomètrie des chinois du sud. Bull. et Mém. Soc. d'Anthrop. de Paris, 11(9):67-90, 1966b.

Ramos, N.; Suazo, I.; Martínez, L. \& Reyes, L. Transverse Face Relations in Chilean Children from Maule Region. Int. J. Morphol., 25(4):703-7, 2007.

Snodell, S. F.; Nanda, R. S. \& Curriel, G. F. A longitudinal cephalometric study of transverse and vertical craniofacial growth. Am. J. Orthod. Dentofacial Orthop., 104(5):471-83, 1993.

Vallejo-Manzur, F.; Perkins, Y.; Varon, J. \& Baskett, P. Andrea Vesalius, the concept of an artificial airway. Resuscitation, 56:3-7, 2003.

Vanrell, J. \& Borborema, M. Identificación Craniométrica, 1953. Disponible en: http://www.periciasforenses.com.br/icraniodo.htm

Dirección para correspondencia:

Prof. María Fabiola Bustamante A.

Facultad de Medicina

Universidad de La Frontera

Temuco

CHILE

Email:fbustama@ufro.cl

Recibido : 22-01-2011

Aceptado: 24-03-2011 